\title{
A TWO-CARDINAL THEOREM
}

\section{SAHARON SHE LAH ${ }^{1}$}

ABSTRACT. We prove the following theorem and deal with some related questions: If for all $n<\omega, T$ has a model $M$ such that $n^{n} \leq\left|Q^{M}\right|^{n}$ $\leq\left|P^{M}\right|<\boldsymbol{\aleph}_{0}$ then for all $\lambda, \mu$ such that $|T| \leq \mu \leq \lambda<\operatorname{Ded}^{*}(\mu)$ (e.g. $\left.\mu=\boldsymbol{\aleph}_{0}, \lambda=2^{\boldsymbol{X}_{0}}\right), T$ has a model of type $(\lambda, \mu)$, i.e. $\left|Q^{M}\right|=\mu,\left|P^{M}\right|=\lambda$.

1. Introduction. We shall deal with first order theories $T$; for simplicity we let $T$ be countable, except in $\$ 3$. It is well known that if $T$ has a model of type $\left(\beth_{\omega}, \boldsymbol{\aleph}_{0}\right.$ ) (i.e. a model $M$ of power $\beth_{\omega}$ with $\left|Q^{M}\right|=\boldsymbol{\aleph}_{0}$ ), then for every $\lambda>\boldsymbol{\aleph}_{0} T$ has a model of type $\left(\lambda, \boldsymbol{\aleph}_{0}\right)$. This is designated by $\left(\beth_{\omega}, \boldsymbol{x}_{0}\right) \rightarrow\left(\lambda, \boldsymbol{\kappa}_{0}\right)$. One may ask the question: For what $\lambda$ does $\left(\boldsymbol{\kappa}_{\omega}, \boldsymbol{\aleph}_{0}\right) \rightarrow$ $\left(\lambda, \boldsymbol{x}_{0}\right)$ ? In particular does $\left(\boldsymbol{x}_{\omega}, \boldsymbol{\aleph}_{0}\right) \rightarrow\left(2 \boldsymbol{\aleph}_{0}, \boldsymbol{\aleph}_{0}\right)$ ? It is of course impossible to ask for more since there is a sentence having a model of type $(\lambda, \mu)$ iff $\boldsymbol{\aleph}_{0} \leq \mu \leq \lambda \leq 2^{\mu}$ (or iff $\boldsymbol{x}_{0} \leq \mu \leq \lambda<\operatorname{Ded}^{*} \mu$ ).

We give a combinatorial lemma which implies $\left(x_{\omega}, \boldsymbol{x}_{0}\right) \rightarrow\left(2 \boldsymbol{x}_{0}, \boldsymbol{x}_{0}\right)$ and seems to be equivalent to it assuming $M A+2 \boldsymbol{\aleph}_{0}>\boldsymbol{\kappa}_{\omega}$. This Lemma still remains an open problem. We finally prove a related two-cardinal theorem (Theorem 1), of interest in its own right, which was stated in the abstract.

\section{Notation.}

Definition 1. A tree is a partially ordered set $(X,<)$ such that for each node $x \in X$ the set of predecessors of $x$ is well ordered by $<$. A branch is a maximal chain. The height of a branch is its order type (always an ordinal).

Definition 2. Let $\mu$ be a cardinal. $\operatorname{Ded}^{*}(\mu)$ is the first power $\lambda$ such that there is no tree with $\leq \mu$ nodes and $\geq \lambda$ branches of the same height. (In this definition we may assume that all trees are subtrees of $\left(\mu^{+} 2,<\right)$, the tree of all $0-1$ sequences of length $<\mu^{+}$, ordered by continuation.)

Received by the editors December 27, 1972 and, in revised form, January 2, 1974.

AMS (MOS) subject classifications (1970). Primary $02 \mathrm{H} 05$.

Key words and phrases. Two-cardinal theorem, finite models.

1I would like to thank Leo Marcus for writing this paper (using notes of my lecture), to thank Papageorgiou for detecting an error, and to thank the referee for a suggestion of reorganization of the paper. 
For example, $\operatorname{Ded}^{*}\left(\boldsymbol{\aleph}_{0}\right)=\left(2^{\boldsymbol{\aleph}_{0}}\right)^{+}$and, in general, $\operatorname{Ded}^{*}(\mu) \leq\left(2^{\mu}\right)^{+}$. See Baumgartner [1] for results about Ded* and Ded (which we shall not even define here); in particular, it is consistent that $\operatorname{Ded}^{*}\left(\boldsymbol{x}_{1}\right)<\left(2 \boldsymbol{x}_{1}\right)^{+}$.

Let $Q$ and $P$ be two unary predicates and $Q^{M}, P^{M}$ their interpretations in the model $M$. We vary from standard notation by letting $(\lambda, \mu)$-model mean a model $M$ with $\left|P^{M}\right|=\lambda,\left|Q^{M}\right|=\mu$.

Our main theorem is thus denoted by $\left\{\left(m_{i}, n_{i}\right): i<\omega\right\} \rightarrow(\lambda, \mu)$ for $\boldsymbol{x}_{0} \leq$ $\mu \leq \lambda<\operatorname{Ded}^{*}(\mu), \kappa_{0}>m_{i}>n_{i}^{i} \geq i^{i}$.

$\eta, \nu$ will denote sequences of zeroes and ones; ${ }^{\alpha} 2$ the set of all $0-1$ sequences of length $\alpha ; l(\eta)$ the length of $\eta ; \eta \hat{\nu}$ the concatenation of $\eta$ and $\nu$; and $\eta \mid \beta$ the initial subsequence of $\eta$ of length $\beta$. Let ${ }^{<\alpha} 2=$ $\bigcup_{\beta<a}^{\beta}$.

3. A two-cardinal theorem. The standard way of proving two-cardinal theorems $\left(\lambda_{0}, \mu_{0}\right) \rightarrow\left(\lambda_{1}, \mu_{1}\right)$ is to find a set of sentences $\Gamma$ such that

(i) if $T$ has a model of type $\left(\lambda_{0}, \mu_{0}\right)$ then $T \cup \Gamma$ is consistent;

(ii) if $T \cup \Gamma$ is consistent then $T$ has a model of type $\left(\lambda_{1}, \mu_{1}\right)$.

Assume w.l.o.g. that $T$ is a theory in a language $L$, and has Skolem functions. We use this method to prove

Theorem 1. If for all $n<\omega$ every finite subset of $T$ has a model $M$ such that $n^{n} \leq\left|Q^{M}\right|^{n} \leq\left|P^{M}\right|<\boldsymbol{x}_{0}$, then for all $\lambda$, $\mu$ such that $|T| \leq \mu \leq \lambda<$ $\operatorname{Ded}^{*}(\mu), T$ has a model of type $(\lambda, \mu)$.

Notice that for $\mu=\boldsymbol{\aleph}_{0}$ the co
$\left(2 \boldsymbol{\aleph}_{0}, \boldsymbol{\aleph}_{0}\right)$ (when $T$ is countable).

Definition 3. Let $\eta_{i}, \nu_{i} \epsilon^{<a} 2$ for $i=1, \cdots, n .\left\langle\eta_{1}, \cdots, \eta_{n}\right\rangle$ and $\left\langle\nu_{1}, \cdots, \nu_{n}\right\rangle$ are similar over $\beta$ if for all $i=1, \ldots, n, l\left(\eta_{i}\right), l\left(\nu_{i}\right) \geq \beta$, $\eta_{i}\left|\beta=\nu_{i}\right| \beta$, and for all $i, j, 1 \leq i<j \leq n, \eta_{i}\left|\beta \neq \eta_{j}\right| \beta$ (and thus $\nu_{i}\left|\beta \neq \nu_{j}\right| \beta$ ).

Definition 4. Let $D$ be a set of $0-1$ sequences. Define

$$
\begin{aligned}
& \Gamma_{L}(D)=\left\{P\left(y_{\eta}\right): \eta \in D\right\} \cup\left\{y_{\eta} \neq y_{\nu}: \eta \neq \nu \in D\right\} \\
& \cup\left\{z_{1}\right.=\tau\left(y_{\eta_{1}}^{-}, \cdots, y_{\eta_{n}}\right) \wedge z_{2}=\tau\left(y_{\nu_{1}}, \cdots, y_{\nu_{n}}\right) \wedge Q\left(z_{1}\right) \\
& \rightarrow z_{1}=z_{2}: \tau \text { is a term in } L, \eta_{i}, \nu_{i} \in D \text { and } \\
&\left.\left\langle\eta_{1}, \cdots, \eta_{n}\right\rangle \text { and }\left\langle\nu_{1}, \cdots, \nu_{n}\right\rangle \text { are similar over some } \beta\right\} .
\end{aligned}
$$

Now, by way of fulfilling part (ii) above it is easy to see

Lemma 1. If $T \cup \Gamma_{L}\left(2^{\omega}\right)$ is consistent and $|T| \leq \mu \leq \lambda<\operatorname{Ded}^{*}(\mu)$, then 
$T$ has a model of type $\left(\lambda, \mu_{1}\right)$, for some $\mu_{1} \leq \mu$. In particular, if $T \cup \Gamma_{L}\left(2^{\omega}\right)$ is consistent and $M$ is the Skolem closure of $\left\{y_{\eta}: \eta \in 2^{\omega}\right\}$, then $M$ is of type $\left(2{ }^{x_{0}}, \boldsymbol{x}_{0}\right)$.

Let us turn now to part (i). We shall list some conditions which are sufficient for proving the consistency of $T \cup \Gamma_{L}\left(2^{\omega}\right)$.

By the compactness theorem, it is enough to show the consistency of $\left.T^{\prime} \cup \Gamma_{L}^{\prime}{ }^{n} 2\right)$ (where the prime on $\Gamma_{L}(D)$ indicates that in the definition of $\Gamma_{L}(D) \tau$ ranges over a finite set of terms of $L$, say $\left\{\tau_{0}, \cdots, \tau_{n_{0}}\right\}$, each having $\leq n_{0}$ variables, and $T^{\prime}$ is a finite subset of $T$ ). This holds because we can replace $T$ by $T_{1}=T \cup\left\{Q\left(c_{i}\right): i<\mu\right\} \cup\left\{c_{i} \neq c_{j}: i<j<\mu\right\}$, the $c_{i}$-new individual constants. $T_{1}$ satisfies the hypothesis of Theorem 1 , and in every model $M$ of it $\left|Q^{M}\right| \geq \mu$. So by the lemma this is sufficient. This must be shown for all $n, n_{0}<\omega$.

Definition 5. Let $M$ be a model, $A$ a subset of $M, \bar{b}, \bar{c} \in M$. Define $\bar{b} \sim \bar{c}(\bmod A)$ if for all $i \leq n_{0}$ and for any presentation of $\tau_{i}, \tau_{i}(\bar{x}, \bar{y})$ (i.e., ordering and identification of the variables of $\tau_{i}$ ), we have for all $\bar{a} \in A$

$$
\tau_{i}(\bar{c}, \bar{a}) \in Q^{M} \vee \tau_{i}(\bar{b}, \bar{a}) \in Q^{M} \Rightarrow \tau_{i}(\bar{c}, \bar{a})=\tau_{i}(\bar{b}, \bar{a})
$$

If $\bar{b}$ is a single-element sequence we simply write $b$.

So clearly if the number of such presentations is $n_{1}$ (so $n_{1}$ depends on $n_{0}$ only), then this equivalence relation has $\leq\left(\left|Q^{M}\right|+1\right)^{k}$ equivalence classes, where $k=|A|^{n}{ }_{n}$.

Claim 1. Let $D$ be a set of $0-1$ sequences of length $n$ and $n-1$ such that no two sequences are comparable (i.e. no one is an initial segment of the other). Assume that the assignment $\left\{y_{\eta} \rightarrow a_{\eta}: \eta \in D\right\}$ satisfies $\Gamma_{L}^{\prime}(D)$. Let $\nu \in D$ be of length $n-1$ and let $d \in P^{M}-\left\{a_{\eta}: \eta \in D\right\}$ be such that $d \sim a_{\nu}\left(\bmod \left\{a_{\eta}: \eta \neq \nu, \eta \in\right.\right.$ $D\})$. Jet $a_{\nu^{\wedge}\langle 0\rangle}=a_{\nu^{\prime}} a_{\nu^{\wedge}\langle 1\rangle}=d$, and $\left.D^{\prime}=(D-\{\nu\}) \cup \nu^{\wedge}\langle 0\rangle, \nu^{\wedge}\langle 1\rangle\right\}$. Then the assignment $\left\{y_{\eta} \rightarrow a_{\eta}: \eta \in D^{\prime}\right\}$ satisfies $\Gamma_{L}^{\prime}\left(D^{\prime}\right)$.

Proof. Let $\left\langle u_{1}, \cdots, u_{n}\right\rangle,\left\langle v_{1}, \cdots, v_{n}\right\rangle$ be similar over some $\beta(\leq n)$, $u_{i}, v_{i} \in D^{\prime}$. We must show

$$
z_{1}=\tau\left(a_{u_{1}}, \cdots, a_{u_{n}}\right) \wedge z_{2}=\tau\left(a_{v_{1}}, \cdots, a_{v_{n}}\right) \wedge Q\left(z_{1}\right) \rightarrow z_{1}=z_{2},
$$

i.e., $\left\langle a_{u_{1}}, \cdots, a_{u_{n}}\right\rangle \sim\left\langle a_{v_{1}}, \cdots, a_{v_{n}}\right\rangle(\bmod \varnothing)$.

If $\beta=n$, we have $u_{i}=v_{i}$ and the result is trivial. If $\beta \leq n-1$, then by the definition of similarity, at most one of the $v_{i}^{\prime}$ 's can be $\nu^{\wedge}\langle 0\rangle$ or $\nu^{\wedge}\langle 1\rangle$; likewise for the $u_{i}$ 's. If none of the $u_{i}^{\prime}$ 's or $v_{i}^{\prime}$ s are $\nu^{\wedge}\langle 0\rangle$ or $\nu^{\wedge}\langle 1\rangle$, then the result holds by our hypothesis. Thus without loss of generality we may 
assume $v_{1} \in\left\{\nu^{\wedge}\langle 0\rangle, \nu^{\wedge}\langle 1\rangle\right\}$. Clearly for $i \neq 1, u_{i}, v_{i} \notin\left\{\nu^{\wedge}\langle 0\rangle, \nu^{\wedge}\langle 1\rangle\right\}$. Now $a_{v_{1}} \sim a_{\nu}\left(\bmod \left\{a_{\eta}: \eta \neq \nu, \eta \in D\right\}\right)$, since either $a_{v_{1}}=a_{\nu}$ or $a_{v_{1}}=d$. Thus $\left\langle a_{v_{1}}, a_{v_{2}}, \cdots, a_{v_{n}}\right\rangle \sim\left\langle a_{\nu}, a_{v_{2}}, \cdots, a_{v_{n}}\right\rangle(\bmod \varnothing)$.

Case 1. $u_{1} \in\left\{\nu^{\wedge}\langle 0\rangle, \nu^{\wedge}\langle 1\rangle\right\}$. Then $\left\langle a_{u_{1}}, a_{u_{2}}, \ldots, a_{u_{n}}\right\rangle \sim$ $\left\langle a_{\nu}, a_{u_{2}}, \cdots, a_{u_{n}}\right\rangle(\bmod \varnothing)$. Clearly $\left\langle\nu, u_{2}, \cdots, u_{n}^{2}\right\rangle$ and $\left\langle u_{n}, v_{2}, \cdots, v_{n}\right\rangle$ are similar over the above $\beta$. And so by the assumption on $\Gamma_{L}^{\prime}(D)$, $\left\langle a_{\nu^{\prime}} a_{u_{2}}, \cdots, a_{u_{n}}\right\rangle \sim\left\langle a_{\nu} a_{v_{2}}, \cdots, a_{v_{n}}\right\rangle(\bmod \varnothing)$. Thus we have $\left\langle a_{u_{1}}, a_{u_{2}}, \cdots, a_{u_{n}}\right\rangle \sim\left\langle a_{v_{1}}, a_{v_{2}}, \cdots, a_{v_{n}}\right\rangle(\bmod \varnothing)$.

Case 2. $u_{1} \notin\left\{\nu^{\wedge}\langle 0\rangle, \nu^{\wedge}\langle 1\rangle\right\}$. Then $\left\langle\nu, v_{2}, \cdots, v_{n}\right\rangle,\left\langle v_{1}, \cdots, v_{n}\right\rangle$, $\left\langle u_{1}, \cdots, u_{n}\right\rangle$ are all similar over $\beta$, so it follows that $\left\langle a_{u_{1}}, \cdots, a_{u_{n}}\right\rangle \sim\left\langle a_{\nu}, a_{v_{2}}, \cdots, a_{v_{n}}\right\rangle \sim\left\langle a_{v_{1}}, a_{v_{2}}, \cdots, a_{v_{n}}\right\rangle(\bmod \varnothing)$. Q.E.D.

Claim 2. In order to show the consistency of $T^{\prime} \cup \Gamma_{L}^{\prime}\left({ }^{n} 2\right)$ for all $n<\omega$ it is sufficient to prove:

For all $m<\omega$ there is a model $M$ of $T^{\prime}$ and a sequence of sets $X_{1} \subset$ $X_{2} \subset \cdots \subset X_{m} \subset P^{M}$ such that for all $i=1, \ldots, m-1$ and all distinct $a_{1}$, $\cdots, a_{m}, a_{m+1} \in X_{i}$, there is $a_{m+1}^{\prime} \in X_{i+1}, a_{m+1}^{\prime} \notin\left\{a_{1}, \cdots, a_{m+1}\right\}$, such that $a_{m+1}^{\prime} \sim a_{m+1}\left(\bmod \left\{a_{1}, \cdots, a_{m}\right\}\right)$.

Proof. This is a corollary of the previous claim by repeated use of it.

Claim 3. Theorem 1 follows from the following combinatorial assertion:

(*) For all $m, k<\omega$ there is $l=l(k, m)<\omega$ such that for all $r<\omega$ : if $F$ is an $m$-place function on a set $A$ of power $|A|=r^{l}$ whose range is subsets of $A$ of power $\leq r$, then there is $B \subset A,|B|=r^{k}$, such that for all distinct $a_{1}, \cdots, a_{m+1} \in B, a_{m+1} \notin F\left(a_{1}, \cdots, a_{m}\right)$.

Proof. We will show that the condition of Claim 2 follows from $(*)$ and the hypothesis of Theorem 1 . Let $l(k, m)$ be as in $(*)$. Define $l_{i}$, for $i=$ $1, \cdots, m-1$, as follows: $l_{1}=1, l_{i+1}=l\left(m, l_{i}\right)$. Choose a model $M$ of $T^{\prime}$ such that $\left|Q^{M}\right| \geq 2,\left|Q^{M}\right| \geq l_{m}, r=\left|Q^{M}\right|^{n_{2}}<\aleph_{0}$, where $n_{2}=2 m^{n_{0}} n_{1}$ and $\left|P^{M}\right| \geq r^{l}$. Let $X_{m}=P^{M}$. For $k=0, \cdots, m-1$ we will define $X_{m-k}$ satisfying the hypothesis of Claim 2 and such that $\left|X_{m-k}\right| \geq r^{l_{m-k-1}}$. Suppose $X_{m-k_{0}}$ satisfying the hypothesis of induction has been found. Let $F$ be the $m$-place function from $X_{m-k_{0}}$ into subsets of $X_{m-k_{0}}$ with less than $r$ elements obtained by letting $F\left(a_{1}, \cdots, a_{m}\right)$ be a complete set of representatives of the equivalence relation $\sim \bmod \left\{a_{1}, \cdots, a_{m}\right\}$. (This 
relation has at most $\left|Q^{M}\right|^{n} 2$ equivalence classes.) Now by $(*)$ there is a set $B=X_{m-k_{0}-1}$ with at least $r^{l_{m-k_{0}-1}}$ elements such that if $a_{1}, \ldots$, $a_{m+1} \in X_{m-k_{0}-1}$ are distinct, then $a_{m+1} \notin F\left(a_{1}, \ldots, a_{m}\right)$, so a choice of $a_{m+1}^{\prime}$ to satisfy the hypothesis of Claim 2 can be made from $F\left(a_{1}, \cdots, a_{m}\right)$.

Now to prove Theorem 1 we need only show

Claim 4. (*) holds.

Remark. Maybe this claim has already appeared in Erdös and Hajnal [3].

Proof. Let $\left\{y_{1}, \cdots, y_{r}\right\}$ be random variables on $A$. What is the probability that $B=\left\{y_{1}, \cdots, y_{r k}\right\}$ will not fulfill the demands of $(*)$ ? It is $\leq$

$$
\begin{aligned}
& \sum_{i_{1}, \cdots, i_{m+1} \leq r}\left[\begin{array}{l}
\text { the probability that } y_{\sigma\left(i_{m+1}\right)} \in F\left(y_{\sigma\left(i_{1}\right)}, \cdots, y_{\sigma\left(i_{m}\right)}\right) \\
\text { for some permutation } \sigma \text { of } i_{1}, \cdots, i_{m+1}
\end{array}\right] \\
& \text { distinct } \\
& +\sum_{1 \leq i \neq j \leq r} k\left[\begin{array}{l}
\text { the probability that } \\
y_{i}=y_{j}
\end{array}\right] \\
& \leq\left(\begin{array}{c}
r^{k} \\
m+1
\end{array}\right) \frac{(m+1) ! r}{r^{l}}+\left(\begin{array}{c}
r^{k} \\
2
\end{array}\right) \frac{1}{r^{l}} \leq \frac{r^{k m+k+1} f(m, k)}{r^{l}}
\end{aligned}
$$

where $f(m, k)$ is some function of $m$ and $k$. So we certainly can choose $l=l(m, k)$ such that the whole expression is $\langle 1$ for all $r\rangle 1$. This means that it is possible to find a suitable set $\left\{y_{1}, \cdots, y_{r k}\right\}$. Q.E.D.

This completes the proof of Theorem 1.

4. Remarks and generalizations. We now turn to the original problem of the consequences of $T$ having a model of type $\left(\boldsymbol{x}_{\omega}, \boldsymbol{x}_{0}\right)$. Consider the following combinatorial assertion.

(**) For all $k, m<\omega$ there is $l<\omega$ such that for any $m$-place function $F$ from $\boldsymbol{\aleph}_{l}$ to the countable subsets of $\boldsymbol{\aleph}_{l}$, there is $A \subseteq \boldsymbol{\aleph}_{l},|A|=\boldsymbol{\aleph}_{k}$, such that for all distinct $a_{1}, \cdots, a_{m}, a_{m+1} \in A, a_{m+1} \notin F\left(a_{1}, \cdots, a_{m}\right)$.

This is the problem mentioned in the introduction; the combinatorial lemma $(* *)$ is known to be true for $m=1$, but for $m>1$ and even $k=0$ it is still an open question. See Hajnal [4].

Theorem 2. If $(* *)$ holds and $T$ has a model of type $\left(\boldsymbol{\aleph}_{\omega}, \boldsymbol{\aleph}_{0}\right)$ then for all $\lambda, \mu$ such that $|T| \leq \mu \leq \lambda<\operatorname{Ded}^{*}(\mu), T$ has a model of type $(\lambda, \mu)$. 
Proof. As in the proof of Theorem 1 it suffices to show that for all $n$ $\Gamma_{L}^{\prime}\left({ }^{n} 2\right)$ is consistent. To see this let $l=l(k, m)$ be as in $(* *)$.

For all $i=1, \ldots, m-1$ define $l_{i}$ as follows: $l_{1}=1, l_{i+1}=l\left(l_{i}, m\right)$. Now let $M$ be a model of $T$ of type $\left(\boldsymbol{\aleph}_{\omega}, \boldsymbol{x}_{0}\right)$. For $i=1, \cdots, m$ we define $A_{i} \subset P^{M}$ by retrograde induction, such that $\left|A_{i}\right|=\kappa_{l_{i}}$ : Choose $A_{m}$ to be any subset of $P^{M}$ of power $\boldsymbol{x}_{l_{m}}$. Now assume that $A_{i+1}$ is defined and for all $a_{1}, \cdots, a_{m} \in A_{i+1}$ let $F\left(a_{1}^{m}, \cdots, a_{m}\right)$ be a set of representatives in $A_{i+1}$ of each equivalence class of $\sim\left(\bmod \left\{a_{1}, \cdots, a_{m}\right\}\right)$. It is not hard to see that there are $\leq \boldsymbol{\aleph}_{0}$ such classes; so $\left|F\left(a_{1}, \cdots, a_{m}\right)\right| \leq \boldsymbol{\aleph}_{0}$, and by $(* *)$ there is $A_{i} \subseteq A_{i+1},\left|A_{i}\right|=\aleph_{l_{i}}$, such that for all distinct $a_{1}, \ldots, a_{m}, a_{m+1} \epsilon$ $A_{i}, a_{m+1} \notin F\left(a_{1}, \cdots, a_{m}\right)$. The sequence $A_{1}, \cdots, A_{m}$ satisfies the requirements of the $X_{i}$ in Claim 2, and so $T \cup \Gamma_{L}^{\prime}\left({ }^{n} 2\right)$ is consistent. Q.E.D.

We may be interested in other theorems of the form: $\left\{\left(m_{i}, n_{i}\right): i<\omega\right\} \rightarrow$ $(\lambda, \mu)$. Vaught's and Chang's two-cardinal theorems (see e.g. [2]) can easily be generalized to this case, but give less than our result (only when $\lambda \leq \mu^{+}$, $\left.\mu=\Sigma_{\kappa<\lambda} \mu^{\kappa}\right)$; Vaught's two cardinal theorem for cardinals far a part generalizes easily to finite hypothesis (using Ramsey's theorem instead of the Erdös-Rado partition theorem) and it cannot be improved. The following remains open (there are, of course, many others):

Question 1. Is our result best possible? That is, does there exist a sentence for which every $n$ has a model $M, \boldsymbol{x}_{0}>\left|P^{M}\right|>\left|Q^{M}\right|^{n},\left|Q^{M}\right| \geq n$, but does not have a $\left(2^{\mu}, \mu\right)$-model for some $\mu$, and even: has a $(\lambda, \mu)$-model iff $\mu \leq \lambda<\operatorname{Ded}^{*}(\mu)$ (assuming for some $\mu$, $\operatorname{Ded}^{*}(\mu) \leq 2^{\mu}$ ).

Conjecture 2. $\left\{\left(m_{i}, n_{i}, k_{i}\right): i<\omega\right\} \rightarrow(\lambda, \mu, \kappa)$ when $m_{i} \geq n_{i}^{i}, n_{i} \geq k_{i}^{i} k_{i}$ $\geq i, \kappa \leq \mu \leq \lambda<\operatorname{Ded}^{*} \kappa$.

Conjecture 3. $\left\{\left(2^{n_{i}}, n_{i}\right): i<\omega\right\} \rightarrow\left(2^{\mu}, \mu\right)\left[n_{i} \geq i\right]$.

The following remarks on the properties of $\Gamma_{L}(D)$ may be useful:

If in Definition 4, we demand only that $\left.k_{i, j}=\min \left\{l: \eta_{i}(l)\right\} \neq \eta_{j}(l)\right\}=$ $\min \left\{l: \nu_{i}(l) \neq \nu_{j}(l)\right\}$, and $\eta_{l}\left(k_{i, j}\right)=\nu_{l}\left(k_{i, j}\right), \eta_{j}\left(k_{i, j}\right)=\nu_{j}\left(k_{i, j}\right)$, we get that the consistency of $T \cup \Gamma_{L}\left({ }^{\omega} 2\right)$ implies $T$ has a $\left(2^{\lambda}, \lambda\right)$-model for every $\lambda$.

It can be shown that the existence of a model of $T$ of type $\left(\lambda, \boldsymbol{x}_{0}\right)$, where $\lambda$ is real-valued measurable, implies the consistency of $\Gamma_{L}\left({ }^{\omega} 2\right)$, even for sentences of $L_{\omega_{1}}, \omega$

Papageorgiou shows that our method gives a positive answer to Conjecture 2 if we strengthen the assumption to: $k_{i} \geq i, n_{i} \geq\left(k_{i}\right)^{i}, m_{i} \geq\left(n_{i}\right)^{\left(n_{i}\right)^{i}}$; and that this generalizes to any finite number instead of three.

It is trivial that if $T$ has a model $M,\left|P^{M}\right| \geq \kappa_{0}>\left|Q^{M}\right|$, then for every 
$\lambda \geq|T|, T$ has a model of type $\left(\lambda,\left|Q^{M}\right|\right)$. Also if for every $n, T$ has a model $M,\left|P^{M}\right| \geq \boldsymbol{\aleph}_{0}>\left|Q^{M}\right| \geq n$, then for every $\lambda \geq \mu \geq|T|, T$ has a model of type $(\lambda, \mu)$. Hence in Theorem 1 we ignore those cases.

On $n$-cardinal theorems see Chang and Keisler [2]. Our result was announced in [5], and $[6, \S 0,(6)$ p. 251$]$. In $[6, \S 0]$ there is a discussion on $n$-cardinal problems.

Added in proof. The main conjecture has been proved and submitted to the Proceedings of the American Mathematical Society.

\section{REFERENCES}

1. J. Baumgartner, Almost-disjoint sets, the dense-set problem, and the partition calculus (to appear).

2. C. C. Chang and H. J. Keisler, Theory of models, North-Holland, Amsterdam, 1973.

3. P. Erdös and A. Hajnal, On the chromatic number of graphs and set-systems, Acta Math. Acad. Sci. Hungar. 17 (1966), 61-99. MR 33 \# 1247.

4. A. Hajnal, Proof of a conjecture of S. Ruziewicz, Fund. Math. 50 (1961), 123-128.

5. S. Shelah, Various results in model theory, Notices Amer. Math. Soc. 19 (1972), A-764. Abstract \# 72T-E 103.

6. - On models with power like orderings, J. Symbolic Logic 37 (1972), $247-267$.

INSTITUTE OF MATHEMATICS, HEBREW UNIVERSITY, JERUSALEM, ISRAEL 